\title{
Reusable chromium-coated quartz crystal microbalance for immunosensing
}

\author{
M.J. Oliver ${ }^{\mathrm{a}, *}$, J. Hernando-García a ${ }^{\mathrm{a}}$ P. Pobedinskas ${ }^{\mathrm{b}}$, K. Haenen $^{\mathrm{b}, \mathrm{c}}$, A. Ríos ${ }^{\mathrm{d}}$, J.L. Sánchez-Rojas ${ }^{\mathrm{a}}$ \\ a Departamento de Ingeniería Eléctrica, Electrónica, Automática y Comunicaciones, E.T.S.I. Industriales, Universidad de Castilla La Mancha, 13071 Ciudad Real, Spain \\ ${ }^{\mathrm{b}}$ Institute for Materials Research (IMO), Hasselt University, 3590 Diepenbeek, Belgium \\ ${ }^{c}$ Division IMOMEC, IMEC vzw, 3590 Diepenbeek, Belgium \\ d Departamento de Química Analítica y Tecnología de los Alimentos, Facultad de Químicas, Universidad de Castilla La Mancha, 13071 Ciudad Real, Spain
}

\section{A R T I C L E I N F O}

\section{Article history:}

Received 7 February 2011

Received in revised form 27 May 2011

Accepted 22 June 2011

Available online 29 June 2011

\section{Keywords:}

Oxidized chromium

Immunosensors

Quartz crystal microbalance

\begin{abstract}
A B S T R A C T
The application of oxidized chromium as a reusable platform for the development of immunosensors is presented. Chromium films were deposited on quartz crystal microbalances to study the affinity interaction between rabbit immunoglobulin $\mathrm{G}(\operatorname{IgG})$ and goat anti-rabbit $\operatorname{IgG}$. A covalent approach, based on the silane chemistry, was followed for the grafting of either the rabbit IgG or the anti-rabbit IgG on the silane-modified chromium surface. Next the differences between the deposition of rabbit IgG on immobilized anti-rabbit IgG and the deposition of anti-rabbit IgG on immobilized rabbit IgG were investigated. The chromium layer could be reused between experiments, after the proper removal of the organic layers with piranha etch, obtaining a high repeatability in the steps of the functionalization protocol.
\end{abstract}

(c) 2011 Elsevier B.V. All rights reserved.

\section{Introduction}

Nowadays there is an increasing interest in the development of biosensors in many different applications [1,2]. These devices consist in a transducer and the biomolecular receptors on the transducer surface that interact with the corresponding analyte. Such interaction is converted by the transducer into a detectable signal. The material of the transducer surface can be of many different types: oxidized silicon [3,4] or H-terminated silicon [5,6], silicon nitride [7,8], GaN and AlN [9], carbon related materials [10,11] or metals such as gold $[12,13]$.

Among the different transduction mechanisms, the quartz crystal microbalance (QCM) is a very well-established technique that allows for a quantitative determination of the process taking place on its surface due to the mass-dependence of its resonance frequency. Such dependence is governed by the Saurbrey equation [14]. It is also important to point out the possibility of measuring the change in the dissipation factor of the QCM after any deposition step and correlate this change with the rheologic properties of the deposited layer [15]. Due to the QCM mass sensitivity, the molecular recognition between antibodies and the corresponding antigens can be easily monitored with this transducer [16,17].

The sensing material for standard QCMs is the gold of the electrodes. The modification of gold is based on the thiol chemistry. The thiol chemistry is very well established due to the ease of

\footnotetext{
* Corresponding author. Tel.: +34 926295300; fax: +34 926295361.

E-mail address: MariaJesus.Oliver@uclm.es (M.J. Oliver).
}

preparation, the uniformity of the films and the large range of available ending groups. Despite of all these advantages, the search of other alternatives to the thiol/gold combination should not be unconsidered, as thiol films are affected by drawbacks, such as limited stability $[18,19]$. In our case, we propose the use of oxidized chromium. This approach would allow us the use of the different functionalization strategies available for oxide surfaces, such as silanes, phosphonates and phosphates, which might overcome the limitations of the thiol chemistry $[20,21]$.

In this work, we deposited chromium thin films on QCMs and, after the oxidation of the chromium layer, studied the interactions between an antibody such as polyclonal goat anti-rabbit immunoglobulin $\mathrm{G}(\operatorname{IgG})$ and the corresponding antigen, rabbit IgG. Oxidized chromium surfaces have already been reported for the study of surfactants adsorption [22] and the deposition of organosilanes [23]. Here we go a step further and demonstrate the reliability of oxidized chromium as a support for immunosensors.

A covalent approach, based on silane chemistry and already used in the literature [24-27], was used for the immobilization of either the antibodies or the antigens. We examined the binding of antigens in solution to the antibody-immobilized layer under varying antigen concentration, as well as the capture of the antibody by the immmobilized antigens. The deposition of antigens on the immobilized antibodies resulted in a saturation monolayer coverage as the antigen concentration was increased. On the contrary, the deposition of antibody on the antigen-immobilized layer presented no saturation.

After the experiments, the chromium-modified QCM was cleaned in order to remove the organic layers and reused again. After reusing the QCM for 6 times, a specific immobilization step of 
the protocol had a standard deviation as low as $1.4 \mathrm{~Hz}$, demonstrating the reusability of oxidized chromium for biosensing purposes.

\section{Materials and methods}

\subsection{Materials}

Rabbit IgG (I8140); polyclonal goat anti-rabbit IgG (R2004); mouse IgG (I5381); anti-mouse IgG (M8642); bovine serum albumin (BSA) (B4287); 3-aminopropyltriethoxysilane (APTES) (A3648); glutaraldehyde (GA) (340855); potassium phosphate monobasic and dibasic were purchased from Sigma-Aldrich; toluene, hydrogen peroxide, sulphuric acid, acetone, 2-propanol were obtained from VWR. Water was purified with a Direct-Q 3 UV Millipore system.

\section{2. $Q C M$}

Gold-terminated QCMs from KSV Instruments were used. The frequency of the first resonance mode was $5 \mathrm{MHz}$ and the active area was $10 \mathrm{~mm}$. $40 \mathrm{~nm}$ thick chromium films were deposited on one side of the QCM by DC-pulsed reactive magnetron sputtering in a home built system [28]. Chromium-coated QCMs are also available commercially [29], not being necessary in that case the chromium deposition step.

The impedance response of the QCM, from the third to the eleventh overtones, was registered using a KSV Instruments QCM-Z500 microbalance system, which determines the resonance frequency and the dissipation factor. The QCM cell was temperature-controlled at $25^{\circ} \mathrm{C}$ thanks to a Peltier element.

\subsection{Elaboration of the sensors}

\subsubsection{Oxidation}

First, the oxidation of chromium was accomplished by immersion of the QCM in fresh piranha etch $\left(\mathrm{H}_{2} \mathrm{SO}_{4}: \mathrm{H}_{2} \mathrm{O}_{2}, 3: 1, \mathrm{v} / \mathrm{v}\right)$ for $5 \mathrm{~min}$. After rinsing in Millipore-Q water, the chromium surface was hydrophilic, as it was completely wet after removal from water. Next the QCM was dried with nitrogen.

\subsubsection{Silanization}

The grafting of APTES was performed as follows. The QCM was immersed in freshly prepared $20 \mathrm{mM}$ APTES solution in toluene for $1 \mathrm{~h}$ in sonication, followed by a thorough cleaning in toluene and isopropanol. The APTES step covered the surface with amineterminated silane organic molecules for the subsequent steps.

\subsubsection{Glutaraldehyde activation}

Next the APTES-modified surface reacted with a $20 \mathrm{mM} \mathrm{GA}$ solution for $1 \mathrm{~h}$, followed by rinsing with water and drying with nitrogen. GA was used as a homo-bifunctional cross-linker between the amine groups of the APTES and the primary amines of the immunoglobulins.

\subsubsection{Biomolecules immobilization}

After the previous step, the QCM was mounted in a cell designed to work in liquid media that allowed the on-line tracking of both the resonance frequency and the dissipation factor of the QCM during the subsequent steps. Once mounted in the cell, the first step was the covalent binding of either the antibody or the antigen, followed by the affinity interaction with the complementary biomolecule. In between the two previous steps, $1 \mathrm{mg} / \mathrm{ml}$ bovine serum albumin (BSA) was used as blocking agent for remaining free binding sites [30]. The rinsing steps between the modification steps were done with PBS. Reactions were considered as complete when equilibrium was reached. Fig. 1 shows the different steps of the process.

\section{Results and discussion}

\subsection{APTES grafting}

The achievement of an APTES-modified chromium surface was checked by measuring the resonance frequency of the QCM after oxidation and after the APTES step. Both measurements were taken in air. A frequency decrease of $15 \mathrm{~Hz}$ was measured. Our result is

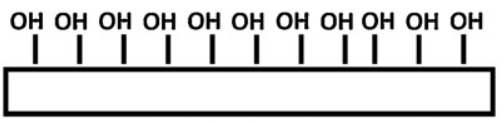

a) Oxidized surface

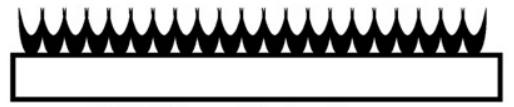

b) APTES-modified surface

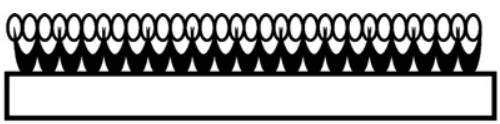

c) Glutaraldehyde deposition

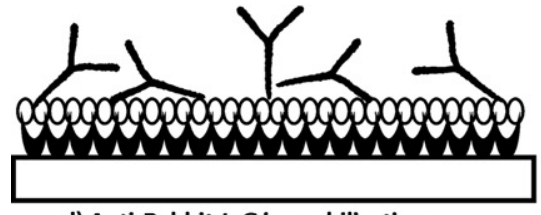

d) Anti-Rabbit-IgG immobilization

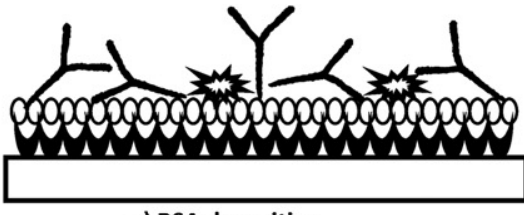

e) BSA deposition

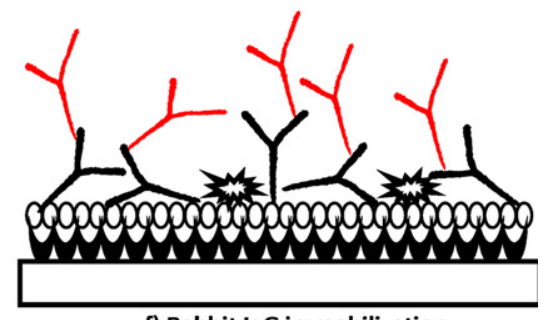

f) Rabbit IgG immobilization

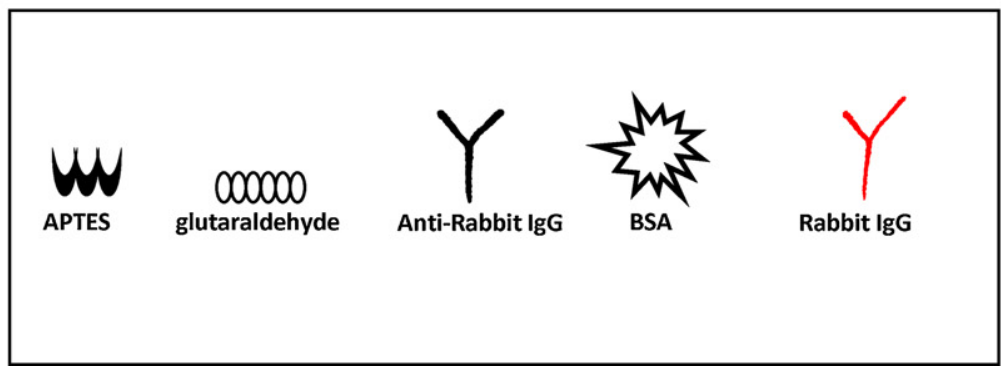

Fig. 1. Steps of the functionalization protocol. 
comparable to already published data. We recall the work by Kurth and Bein [31]. These authors measured a frequency shift of $-48 \mathrm{~Hz}$ for the adsorption of APTES on oxidized aluminum-coated $9 \mathrm{MHz}$ QCM and such a shift was attributed to the formation of nearly one monolayer of APTES. The shift of $-48 \mathrm{~Hz}$ for a $9 \mathrm{MHz}$ QCM corresponds to a surface mass density of $259 \mathrm{ng} / \mathrm{cm}^{2}$. In our case, the frequency shift is $-15 \mathrm{~Hz}$, and, assuming monolayer coverage, such a value corresponds to a surface density of $265 \mathrm{ng} / \mathrm{cm}^{2}$ for a $5 \mathrm{MHz}$ QCM.

Gambinossi et al. [32] also reported the frequency shift for the chemisorption of (3-aminopropyl)trimethoxysilane (APTMS) molecules on silicon oxide. This work and our experiments are done with the same QCM, so the frequency shifts can be directly compared. According to this work, a densely packed monolayer of APTMS gives a frequency shift of about $-10 \mathrm{~Hz}$.

Moreover, in order to be sure that this value was due to the grafting process, we repeated the same APTES process on a goldterminated QCM. No grafting of APTES is expected on the goldterminated QCM, as there are no hydroxyl groups on its surface. We did not observe any frequency shift for the gold-terminated QCM after the APTES process, confirming that the frequency shift we measured for the chromium-coated QCM was related to the APTES grafting.

\subsection{Rabbit IgG on anti-rabbit IgG-immobilized QCM}

Fig. 2 shows the different steps of rabbit IgG deposition on antirabbit IgG-immobilized QCM after the glutaraldehyde activation. All these steps were registered on-line in liquid, monitoring both the resonance frequency shift and the dissipation factor change.

\subsubsection{Anti-rabbit IgG immobilization}

First PBS was introduced into the QCM cell and, once the equilibrium was reached, a solution of anti-rabbit $\operatorname{IgG}(100 \mu \mathrm{g} / \mathrm{ml})$ was allowed to react with the functionalized quartz crystal. The frequency shift after the immobilization of the anti-rabbit IgG layer was $-34 \pm 1.4 \mathrm{~Hz}(n=6)$, equivalent to a mass surface density of $602 \mathrm{ng} / \mathrm{cm}^{2}$, according to the Sauerbrey equation. Depending on the IgG orientation, the coverage of a densely packed monolayer of anti-rabbit IgG molecules may vary between about $200 \mathrm{ng} / \mathrm{cm}^{2}$ $\left(-11 \mathrm{~Hz}\right.$, side-on orientation) and $400 \mathrm{ng} / \mathrm{cm}^{2}(-22 \mathrm{~Hz}$, end-on orientation) [16,33], values that are below the measured shift. So a multilayer of anti-rabbit IgG molecules might be expected.

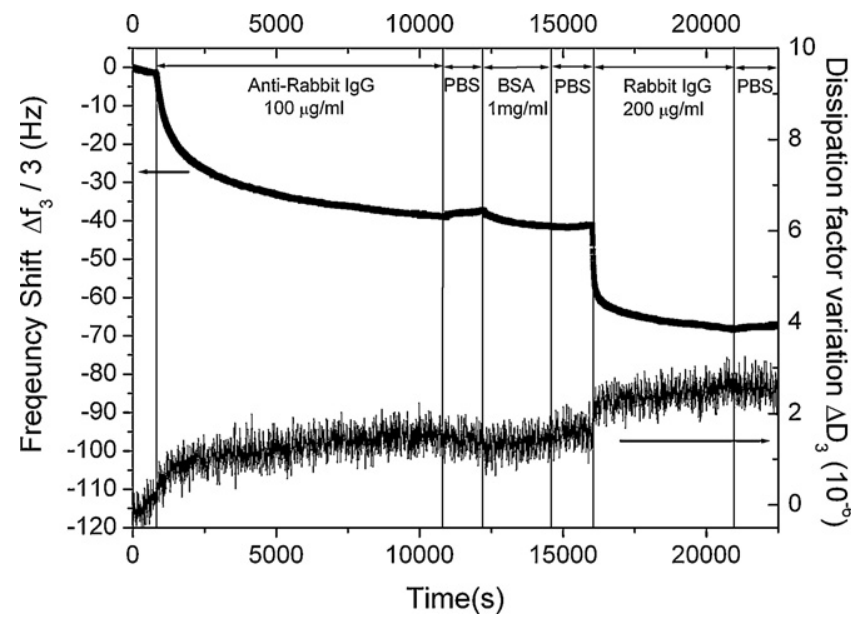

Fig. 2. Resonance frequency shift of the third overtone normalized by 3 and dissipation factor variation of the same overtone for the GA-APTES-modified $\mathrm{Cr}$-deposited QCM versus time during the following steps: (i) $100 \mu \mathrm{g} / \mathrm{ml}$ anti-rabbit IgG solution in PBS, (ii) $1 \mathrm{mg} / \mathrm{ml} \mathrm{BSA}$, and (iii) $200 \mu \mathrm{g} / \mathrm{ml}$ rabbit IgG solution in PBS.
However, the uptake of water by the layer of biomolecules has already been proposed in the literature as an additional source of mass to be taken into account when evaluating biomolecules deposition in liquid media by QCM measurements [15,34]. Depending on the biomolecule under study, the coupled water may vary from $30 \%$ [35] to $90 \%$ [33] of the deduced total mass. So, at this point, it is difficult to estimate the real coverage of this layer. It should also be noted that the application of the Sauerbrey equation requires a thin rigid layer on the QCM. For nonrigid dissipative viscoelastic films, the previous equation fails. In order to estimate its applicability, the variation of the dissipation factor $(\Delta D)$ of the QCM has to be tracked while depositing the layer. $\Delta D$ values about or below $2 \times 10^{-6}$ guarantee the use of the Sauerbrey equation [15]. Higher values indicate a flexible layer with a high water content uptake $[15,36]$. Fig. 2 shows that the $\Delta D$ for the anti-rabbit IgG layer is below $2 \times 10^{-6}$, therefore, within the required range to apply the Sauerbrey equation.

\subsubsection{BSA deposition}

After the anti-rabbit IgG binding step, a solution of BSA was introduced. The BSA step resulted in a very small frequency shift, about $-3 \mathrm{~Hz}$. This might be due to either a non-homogeneous coverage of the former layer that exposed free sites or an uncontrolled physisorption process of the BSA molecules on the anti-rabbit IgG layer. This small shift confirms that the anti-rabbit IgG layer is covering nearly all the active area of the QCM [37].

\subsubsection{Rabbit IgG/anti-rabbit IgG interaction}

For the final step, corresponding to the antigen-antibody interaction, $200 \mu \mathrm{g} / \mathrm{ml}$ rabbit IgG solution in PBS were added and the resulting frequency shift was $-25 \mathrm{~Hz}$. Here the $\Delta D$ is close to $1 \times 10^{-6}$.

In order to get more information about the interaction antibody-antigen, the dependence of the frequency shift on the concentration of rabbit IgG that interacts with the anti-rabbit IgGmodified QCM was studied. Fig. 3 shows the results. The data points correspond to the average of repeated experiments. And the value of each experiment is the normalized frequency shift of the different overtones. The error bars indicate the standard deviation of the repeated experiments. It is worth mentioning that the $\Delta D$ was below $2 \times 10^{-6}$ for these experiments. There are two clear regions: a linear region up to $20 \mu \mathrm{g} / \mathrm{ml}$ and a saturation region above the

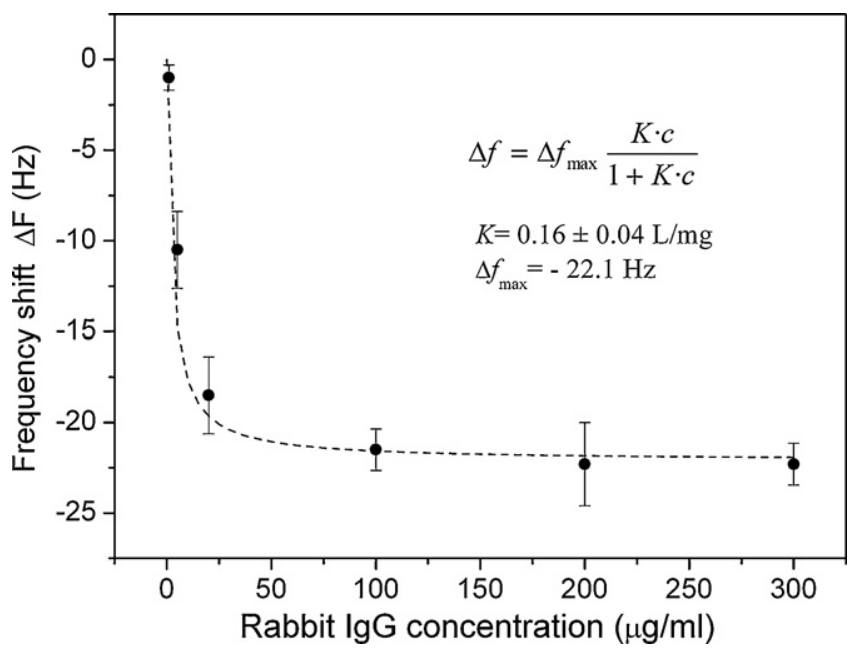

Fig. 3. Frequency shift due to the interaction of rabbit IgG with anti-rabbit IgGmodified QCM. The dashed curve is the fitting to a Langmuir-type adsorption model. The data points are the frequency shift average of repeated experiments. The error bars represent the standard deviation of the repeated experiments. The number of experiments was 3 for the three higher concentrations, and 2 for the rest. 
previous concentration. As can be seen, the saturation value corresponds to about $-23 \mathrm{~Hz}$. This type of behaviour is commonly found in the field of immunosensors when studying antibody-antigen affinity and resembles a Langmuir-type adsorption model [38-40]. The dashed line of Fig. 3 is the fitting of the experimental data to the equation governing the Langmuir adsorption model:

$\Delta f=\Delta f_{\max } \frac{K c}{1+K c}$

where $\Delta f$ is the measured frequency shift in Hertz measured at each IgG concentration, $\Delta f_{\max }$ is the frequency shift at saturation, $c$ is the rabbit IgG concentration in solution and $K$ is the affinity constant. $\Delta f_{\max }=-22.1 \mathrm{~Hz}$ and $K=0.16 \pm 0.04 \mathrm{~L} / \mathrm{mg}$, comparable to previous results on gold surfaces [40], were obtained from the fitting. As a result, the frequency shift saturation of $-23 \mathrm{~Hz}$ is expected to correspond to the coverage of rabbit IgG required to saturate the active binding sites present on the anti-rabbit IgG layer. This value compares well with the frequency shift expected for a monolayer of IgG molecules with end-on Y orientation. Similar results were obtained before. For example, He et al. [41], reported the reaction of human IgG on anti-human IgG adsorbed on gold-terminated QCM. After successive additions, the frequency shift saturated and the final frequency shift was about $-150 \mathrm{~Hz}$ for a total IgG concentration of $167 \mu \mathrm{g} / \mathrm{ml}$. The fundamental resonance frequency and the active area for this work were $9 \mathrm{MHz}$ and a $6.5 \mathrm{~mm}$ diameter circle, respectively. So, the corresponding frequency shift for the QCM used in our experiments would be $-19 \mathrm{~Hz}$, comparable to the value of $-23 \mathrm{~Hz}$ we reported.

The specificity of the interaction was also studied using mouse $\operatorname{IgG}$, a non-specific antigen. The frequency shift for $20 \mu \mathrm{g} / \mathrm{ml} \mathrm{specific}$ rabbit IgG and for $20 \mu \mathrm{g} / \mathrm{ml}$ non-specific mouse $\operatorname{IgG}$ were $-20 \mathrm{~Hz}$ and below the resolution of the system, respectively. Fig. 4 shows the results.

Equally interesting is to mention that the same chromiumcoated QCM was used for 6 experiments in Fig. 3, ranging from the lowest to the highest concentration. For each concentration, all the protocol steps were repeated, including the initial immersion in piranha etch to regenerate and clean the oxidized chromium surface. This allowed us to check the reproducibility of the step corresponding to the immobilization of anti-rabbit IgG on the GAAPTES-modified QCM, as this step was the same for all the 6 experiments. The average frequency shift of this step was $-34 \mathrm{~Hz}$ with a standard deviation of $1.4 \mathrm{~Hz}$ as Fig. 5 shows. This result

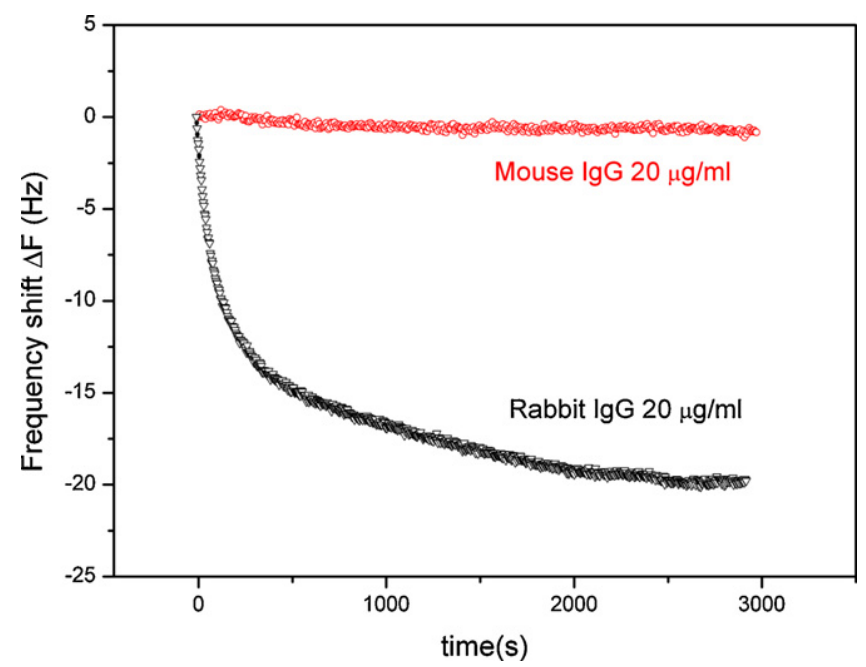

Fig.4. Specificity of the antibody-antigen interaction. Response of $20 \mu \mathrm{g} / \mathrm{ml}$ specific rabbit IgG and $20 \mu \mathrm{g} / \mathrm{ml}$ non-specific mouse IgG on anti-rabbit IgG-immobilized QCM.

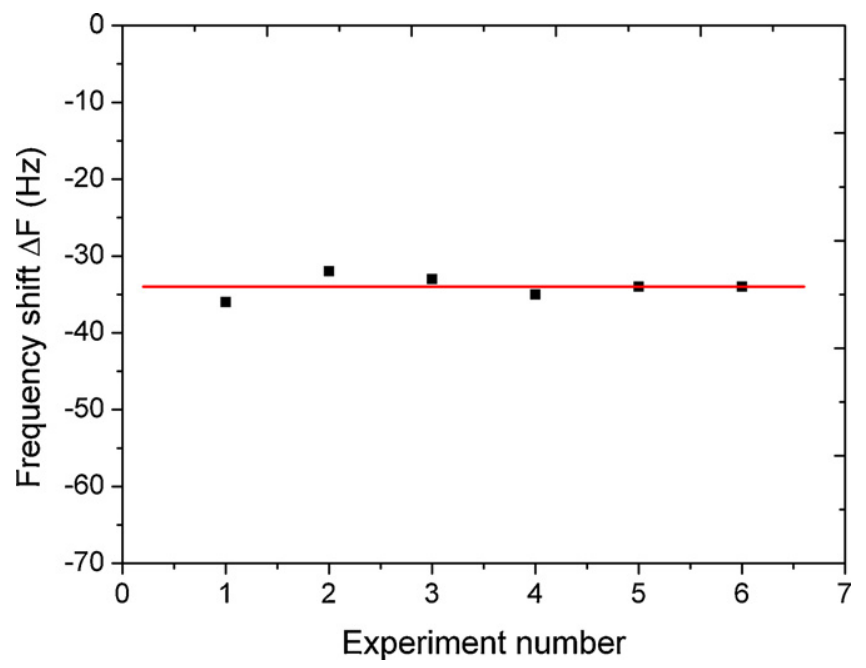

Fig. 5. Repeatability of the immobilization of $100 \mu \mathrm{g} / \mathrm{ml}$ anti-rabbit IgG on GAAPTES-modified chromium-coated QCM.

demonstrates the reliability of oxidized chromium as reusable support for biosensing in liquid media.

\subsection{Anti-rabbit IgG on rabbit IgG-immobilized QCM}

We also used a chromium-deposited QCM to study the interaction of anti-rabbit IgG on an immobilized layer of rabbit IgG, i.e. the order in which the biomolecules were deposited was inverted with respect to Figs. 2 and 3. The rest of the steps were all the same. The same QCM was used for the different concentrations, after regeneration with piranha etch. The step corresponding to the covalent link of the rabbit IgG to the GA-APTES-modified QCM was done using a $50 \mu \mathrm{g} / \mathrm{ml}$ concentration in PBS and the frequency shift was $-23 \pm 3.6 \mathrm{~Hz}(n=6)$. The deposition of BSA gave the same results as the first approach. The frequency shift as a function of the antirabbit IgG concentration that reacts with the rabbit IgG-modified QCM can be seen in Fig. 6. Surprisingly, the obtained behaviour differs from that explained in Section 3.2. We could expect a saturation of the response as the antibody concentration increases. However, there is no saturation plateau but a decrease in the resonance frequency shift up to concentrations of $200 \mu \mathrm{g} / \mathrm{ml}$. An explanation to

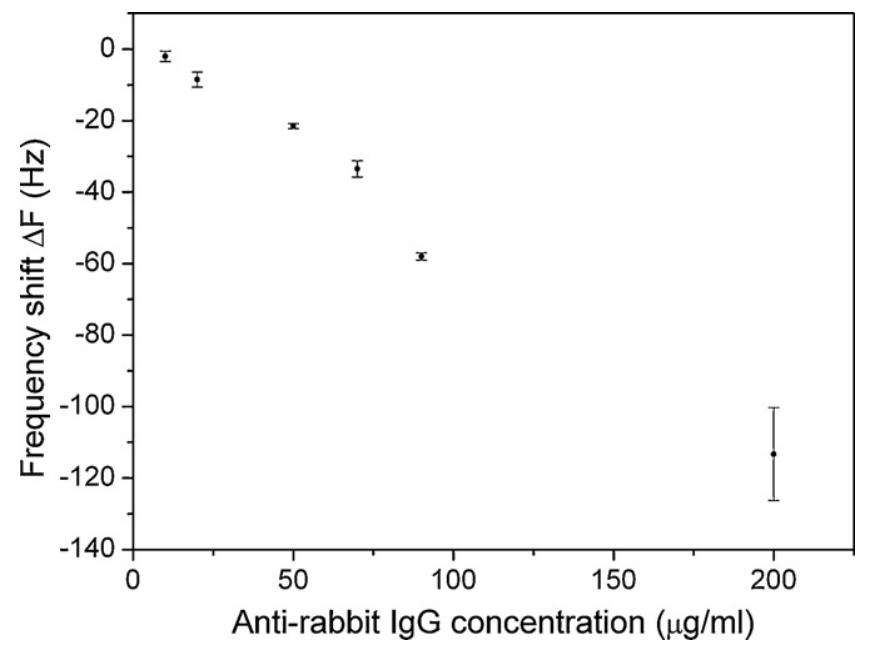

Fig. 6. Frequency shift due to the interaction of anti-rabbit IgG with rabbit IgG-modified QCM. The data points are the frequency shift average of repeated experiments. The error bars represent the standard deviation of the repeated experiments. The number of experiments was 3 for the two higher concentrations, and 2 for the rest. 
this behaviour could be related to the nature of the biomolecules used. The rabbit IgG, i.e. the antigen in our experiments, is a large molecule with many different binding sites (epitopes) for the antibodies [42]. Antibodies can be either monoclonal or polyclonal. Monoclonal antibodies only recognize a specific epitope of the antigen. And polyclonal antibodies recognize all the different epitopes. In our case, the anti-rabbit IgG antibody is polyclonal. Therefore, the numerous epitopes of the rabbit IgG could be targeted. It also has to be mentioned that each anti-IgG molecule has two binding sites for the epitopes of the antigen [42].

So we can conclude that immobilized anti-IgG molecules present no more than two binding sites for the dissolved IgG antigens. And immobilized IgG molecules show several binding sites (epitopes) for the polyclonal anti-IgG antibodies. Therefore, assuming a random orientation, a higher number of binding sites might be present on the surface modified with rabbit IgG molecules. In consequence, a higher amount of anti-IgG molecules will be required to achieve saturation, and this might be why there is no evidence of saturation in Fig. 6 for the range of anti-IgG concentrations under investigation.

Another important feature to point out is the value of the dissipation factor variation $\Delta D$ in Fig. 6. The value of $\Delta D$ is higher than $2 \times 10^{-6}$ for concentrations above $70 \mu \mathrm{g} / \mathrm{ml}$. For example, $\Delta D$ is about $3 \times 10^{-6}$ and $6 \times 10^{-6}$ for $90 \mu \mathrm{g} / \mathrm{ml}$ and $200 \mu \mathrm{g} / \mathrm{ml}$, respectively. This trend in $\Delta D$ might correspond to a higher water content uptake by the increasing coverage in anti-IgG. In addition, such values also reveal a viscoelastic film and the corresponding frequency shifts $(-58 \mathrm{~Hz}$ and $-113 \mathrm{~Hz}$ for $90 \mu \mathrm{g} / \mathrm{ml}$ and $200 \mu \mathrm{g} / \mathrm{ml}$ over estimate the real added mass [43]. It is also worth noting the standard deviation of the frequency shift at $200 \mu \mathrm{g} / \mathrm{ml}$. This error is higher than for the lower concentrations and this could be related to the importance of coupled water to the measured frequency shift and the variability in this water uptake.

Similar experiments were carried out in-house on silicon-oxide modified quartz crystals microbalances and the same trend, i.e. no saturation was observed. The main drawback was the need of using a new silicon oxide QCM for each concentration, as we did not get reproducible results after regeneration with piranha etch.

\section{Conclusions}

We report on the application of oxidized chromium thin films as a reusable platform for the development of immunosensors. Chromium films were deposited on QCM to study the biomolecular recognition between rabbit IgG and the corresponding goat anti-rabbit IgG. A covalent approach, based on the silane chemistry, was followed for the grafting of either the rabbit IgG or the antirabbit IgG on the surface, with the subsequent deposition of the complementary biomolecule. The chromium surface allowed us to investigate the differences between depositing rabbit IgG on immobilized anti-rabbit IgG and anti-rabbit IgG on immobilized rabbit IgG. The first approach resulted in a deposition curve terminated by a saturation plateau. By contrast the second approach exhibited no saturation. The chromium layer could be reused between experiments, after the proper removal of the organic layers with piranha etch, obtaining a high repeatability in the steps of the functionalization protocol. These results demonstrate the reliability of oxidized chromium thin films as a reusable support for the analysis of biomolecules interactions and as a realistic alternative to other metals.

\section{Acknowledgements}

This work was supported by Junta de Comunidades de Castilla La Mancha Project Number PCC08-0015-0722, the Spanish Ministerio de Educación y Ciencia Project Number DPI2009-07497, the Hasselt University Special Research Fund (UHasselt-BOF), and the Belgian Science Policy IAP-Project P6/42. M.J. Oliver acknowledges financial support from BES-2007-16399.

\section{References}

[1] Vo-Dinh, B. Cullum, Fresenius Journal of Analytical Chemistry 366 (2000) 540-551.

[2] P.D. Patel, Trends in Analytical Chemistry 21 (2002) 96-115.

[3] R. Lenigk, M. Carles, N.Y. Ip, N.J. Sucher, Langmuir 17 (2001) 2497-2501

[4] A. Subramanian, S.J. Kennel, P.I. Oden, K.B. Jacobson, J. Woodward, M.J. Doktycz, Enzyme Microbial Technology 24 (1999) 26-34.

[5] B.R. Hart, S.E. Létant, S.R. Kane, M.Z. Hadi, S.J. Shields, J.G. Reynolds, Chemical Communications 322-323 (2003).

[6] R. Boukherroub, Current Opinion in Solid State and Materials Science 9 (2005) 66-72.

[7] M. Manning, G. Redmond, Langmuir 21 (2005) 395-402.

[8] H. Gao, R. Luginbtihl, H. Sigrist, Sensors and Actuators B 38-39 (1997) 38-41.

[9] B. Baur, G. Steinhoff, J. Hernando, O. Purrucker, M. Tanaka, B. Nickel, M. Stutzmann, M. Eickhoff, Applied Physics Letters 87 (2005) 263901.

[10] A. Härtl, E. Schmich, J.A. Garrido, J. Hernando, S.C.R. Catharino, S. Walter, P. Feulner, A. Kromka, D. Steinmüller, M. Stutzmann, Nature Materials 3 (2004) 736-742.

[11] M. Díaz-González, D. Hernández-Santos, M.B. González-García, A. Costa-García, Talanta 65 (2005) 565-573.

[12] S. Dong, J. Li, Bioelectrochemistry and Bioenergetics 42 (1997) 7-13.

[13] E. Ostuni, L. Yan, G.M. Whitesides, Colloids and Surfaces B: Biointerfaces 15 (1999) 3-30.

[14] G. Sauerbrey, Zeitschrift Fur Physik 155 (1959) 206-222.

[15] F. Höök, B. Kasemo, T. Nylander, C. Fant, K. Sott, H. Elwing, Analytical Chemistry 73 (2001) 5796-5804.

[16] F. Caruso, E. Rodda, D. Neil Furlon, Journal of Colloid and Interface Science 178 (1996) 104-115.

[17] S. Kurosawa, J.-W. Park, H. Aizawa, S.-I. Wakida, H. Tao, K. Ishihara, Biosensors and Bioelectronics 22 (2006) 473-481.

[18] G.G. Baralia, A.-S. Duwez, N. Bernard, A.M. Jonas, Langmuir 21 (2005) 6825-6829.

[19] J.B. Schlenoff, M. Li, H. Ly, Journal of the American Chemical Society 117 (1995) 12528-12536.

[20] G.G. Baralia, A. Pallandre, B. Nysten, A.M. Jonas, Nanotechnology 17 (2006) 1160-1165.

[21] M. Dubey, T. Weidner, L.J. Gamble, D.G. Castner, Langmuir 26 (2010) $14747-14754$

[22] F. Caruso, T. Serizawa, D. Neil Furlong, Y. Okahata, Langmuir 11 (1995) 1546-1552.

[23] I. Anac, T.J. McCarthy, Journal of Colloid and Interface Science 331 (2009) $138-142$.

[24] H. Berney, P. Roseingrave, J. Alderman, W. Lane, J.K. Collins, Sensors and Actuators B 44 (1997) 341-349.

[25] Z.-H. Wang, G. Jin, Colloids and Surfaces B: Biointerfaces 34 (2004) 173-177.

[26] M. Varshneya, P.S. Waggonera, R.A. Montagnab, H.G. Craighead, Talanta 80 (2009) 593-599.

[27] N. Nugaeva, K.Y. Gfeller, N. Backmann, M. Düggelin, H.P. Lang, H.-J. Güntherodt, M. Hegner, Microscopy Microanalysis 13 (2007) 13-17.

[28] V. Mortet, M. Nesládek, K. Haenen, A. Morel, M. D’Olieslaeger, M. Vaněček, Diamond Related Materials (2004) 1120-1124, 13/4-8.

[29] www.qsense.com.

[30] R. Danczyk, B. Krieder, A. North, T. Webster, H. HogenEsch, A. Rundell, Biotechnology and Bioengineering 84 (2003) 215-223.

[31] D.G. Kurth, T. Bein, Langmuir 9 (1993) 2965-2973.

[32] F. Gambinossi, L. Lorenzelli, P. Baglioni, G. Caminati, Colloids and Surfaces A: Physicochemical and Engineering Aspects 321 (2008) 87-93.

[33] J. Buijs, J.W.Th. Lichtenbelt, W. Norde, J. Lyklema, Colloids and Surfaces B: Biointerfaces 5 (1995) 11-23.

[34] J. Rickert, A. Brecht, W. Gö1pel, Analytical Chemistry 69 (1997) 1441-1448.

[35] F. Höök, M. Rodahl, P. Brzezinski, B. Kasemo, Langmuir 14 (1998) 729-734.

[36] S. Paul, D. Paul, T. Basova, A.K. Ray, Journal of Physical Chemistry C 112 (2008) 11822-11830.

[37] C. Zhou, J.-M. Friedt, A. Angelova, K.-H. Choi, W. Laureyn, F. Frederix, L.A. Francis, A. campitelli, Y. Engelborghs, G. Borghs, Langmuir 20 (2004) 58705878.

[38] G. Sakai, S. Nakata, T. Uda, N. Miura, N. Yamazoe, Electrochimica Acta 44 (1999) 3849-3854.

[39] N. Soh, T. Tokuda, T. Watanabe, K. Mishima, T. Imato, T. Masadome, Y. Asano, S. Okutani, O. Niwa, S. Brown, Talanta 60 (2003) 733-745.

[40] E. Briand, M. Salmain, C. Compère, C.-M. Pradier, Biosensors and Bioelectronics 22 (2007) 2884-2890.

[41] H. He, Q. Xie, Y. Zhang, S. Yao, Journal of Biochemical and Biophysical Methods 62 (2005) 191-205.

[42] R. Cowan, P. Anne Underwood, Journal of Theoretical Biology 132 (1988) 319-335.

[43] S. Boujday, A. Bantegnie, E. Briand, P.-G. Marnet, M. Salmain, C.-M. Pradier, Journal of Physical Chemistry B 112 (2008) 6708-6715. 